\title{
Dimensional interactions in vibrotactile information processing
}

\author{
BRUCE TAYLOR \\ Princeton University, Princeton, New Jersey 08540
}

\begin{abstract}
The effect of irrelevant dimensional variation on the processing of vibrotactile stimuli was measured. Six observers performed a speeded classification task with stimuli varying along the dimensions of pitch and loudness. Choice reaction times were obtained for stimuli differing on one dimension alone, on two correlated dimensions, or on two orthogonally varied dimensions. Compared to one-dimension performance, reaction times were faster in the correlated condition and slower in the orthogonal condition. In general, these findings agreed with similar experiments in other modalities, with the exception that the effects in this study tended to be stronger for cases in which loudness, rather than pitch, was the relevant dimension. The results are explained in terms of the integrality of pitch and loudness and of the relative discriminability of dimensions.
\end{abstract}

Attempts to employ the skin as an alternate communication channel have led to the construction of a wide variety of devices that deliver either pictorial or coded information to the skin (Farrell, 1950; Geldard, in press). One prominent feature of most of the tactile communication systems that have been developed so far is their simplicity. Pictorial displays, which convey a spatial representation of information, rely almost exclusively on the dimension of locus. A similar situation exists with coded display systems. Even in multidimensional codes, simplicity has been the rule. Typically; the elements of these codes are stimuli that differ along one, and only one, dimension. An error in discrimination along any dimension transforms the information carried by the entire element. While the logical and practical appeal of a parsimonious approach is obvious, it is also clear that the auditory and visual codes for which cutaneous sytems are intended to substitute are often characterized by a great deal of structural redundancy. It seems possible, therefore, that cutaneous languages might also benefit from the addition of stimulus changes beyond those logically necessary for making the desired discriminations.

Over the past few decades. the effects of stimulus redundancy have been studied extensively in the visual and auditory modalities. Yet, as Garner (1972) has observed, these studies have often produced conflicting results, and there is still considerable uncertainty as to the conditions under which redundancy can actually improve information processing. Recently, Garner (1974) has summarized the data

The experimental work reported in this paper was supported by Grant NS-04755, from the National Institutes of Health. The author wishes to thank Dr. Wendell R. Garner for valuable suggestions in regard to the revision of this manuscript. from a wide range of experiments on this problem. His conclusion is that the effects of stimulus redundancy upon perceptual performance depend on the nature of the stimulus dimensions inyolved and the relationships that exist among those dimensions. Specifically, he distinguishes between integral and separable dimensions. Although the concept of integrality is defined by a variety of converging experimental operations, it essentially describes dimensions that join to form phenomenologically unitary stimuli that are difficult to analyze into their distinct components. One important characteristic of integral dimensions is that, in similarity scaling experiments, they are found to combine according to a Euclidean metric (Garner, 1974, p. 102). Integral stimulus dimensions are contrasted with separable dimensions, which are more easily analyzable, can be attended to and processed individually, and combine according to a city-block metric. Garner and his colleagues have shown the usefulness of this distinction in understanding the results of a variety of information processing studies. In general, stimulus discrimination and classification tasks have indicated that dimensional redundancy can improve performance with integral dimensions, but has essentially no effect upon performance with separable dimensions.

The aim of the present study was to apply these findings in an attempt to assess the effect of simple forms of redundancy upon the processing of vibrotactile stimuli. The experimental paradigm adopted for this purpose was a speeded classification task that has been used extensively by Garner (1974) and his associates, and others. The simplest form of this design involves the generation of a set of four stimuli from the orthogonal combination of two levels on each of two different stimulus dimensions. The ob- 
server's task is to classify either two or all four of these stimuli on the basis of one of the stimulus dimensions, called the relevant dimension. The question of interest is the effect of manipulations of the other dimension (the irrelevant dimension) upon the speed with which the classifications can be made.

The set of stimuli to be classified in any particular block of trials is selected so that the irrelevant dimension is combined with the relevant dimension in one of the following ways: (1) The irrelevant dimension is held constant over both levels of the relevant dimension, (2) each level of one dimension is completely correlated with one level of the other dimension, or (3) the irrelevant dimension is varied independently of the relevant dimension. Garner (1974) has labeled the stimulus sets resulting in these three cases one-dimension, correlated, and orthogonal, respectively. The classification task performed with the one-dimension set is considered to be the control condition, indicating the speed with which sorting can be done on the basis of differences along either of the two dimensions separately. With the correlated set, the effect of redundancy can be measured, since the information carried by the relevant dimension is also contained in the irrelevant dimension. Lastly, the classification of the orthogonal stimulus set addresses the converse problem of selective attention, i.e., the degree to which irrelevant stimulus variations can be ignored.

The present study employed vibrotactile stimuli varying along the dimensions of pitch and loudness. The experimental design was similar to that outlined above, with the exception that four, rather than two, levels of loudness were used to generate the total stimulus set. However, since only two loudness levels were used at any given time, the tasks associated with each of the experimental conditions were essentially the same as those of previous visual and auditory studies.

\section{METHOD}

\begin{abstract}
Observers
Six observers were employed in the present study, all of whom were recruited from among laboratory and support personnel. Two of the observers were highly experienced in observing cutaneous stimuli. Another observer was well trained in a variety of psychophysical tasks, and had some experience in working with cutaneous stimuli. The remaining three observers were essentially naive with respect to psychophysical observation. All observers participated in a 2 -h practice session that familiarized them with each of the three different test procedures of the experiment.
\end{abstract}

\footnotetext{
Apparatus

The apparatus was designed to deliver vibrotactile stimuli of desired frequency, intensity, and duration to a Goodmans V-47 vibrator, and to measure reaction times to these stimuli. The signal sources were a pair of Hewlett-Packard 200AB audio oscillators, set at 80 and $300 \mathrm{~Hz}$, respectively. The two channels were separately attenuated, then gated by a Tally tape reader that
}

determined the type and order of stimuli delivered for any given block of trials. A single output leading from the tape reader was then amplified and gated by a Grason-Stadler Type E 3382B electronic switch to yield a signal with a rise time of $10 \mathrm{msec}$. The signal was further amplified and finally led to the vibrator. Timing of the stimuli was determined by a set of Tektronix 160 series pulse generators, which controlled the stepping rate of the tape reader.

The vibrator, with a circular contactor $1 \mathrm{~cm}$ in diameter and a static surround $2.5 \mathrm{~cm}$ in diameter, was suspended on a counterbalanced support. The contactor and surround protruded through the bottom of a padded platform on which the observer's left arm was positioned, and rested against the index finger with a static force of about $20 \mathrm{~g}$. The observer's right hand rested on a second platform containing the two response buttons.

Reaction times were measured to the nearest millisecond by a Hewlett-Packard 5512A electronic counter, and recorded, along with the button choice, by the experimenter. Throughout the experiment, the observer wore a pair of sound-attenuating headphones. To mask further the audible tones generated by the vibrator, white noise was presented to the observer through a speaker located near the stimulus platform.

\section{Procedure}

The test procedure involved three separate steps that were performed with each of the observers. First, absolute threshold for the $80-\mathrm{Hz}$ signal was obtained by the method of limits. Stimulus bursts of $500 \mathrm{msec}$ were presented with an interstimulus interval of $1,500 \mathrm{msec}$. Intensity was changed in 1-dB steps. Threshold was taken as the median coil voltage of four ascending and four descending runs administered in a counterbalanced order. Second, intensity levels of $22,28,34$, and $40 \mathrm{~dB}$ relative to this threshold were calculated, and the observer then matched the subjective magnitude of a $300-\mathrm{Hz}$ signal to that of each of these four stimuli in turn by the method of adjustment. In this procedure, alternating 500 -msec bursts of 80 - and $300-\mathrm{Hz}$ stimuli were presented with an interstimulus interval of $500 \mathrm{msec}$. Intensity of the $300-\mathrm{Hz}$ signal was varied in $1-\mathrm{dB}$ steps until the observer was satisfied that the two stimuli were equal in loudness. By this method, there were generated for each observer, four pairs of stimuli, within which the only difference was one of pitch, and between which there were successive loudness differences of roughly equal psychological magnitude. Using only one pair of adjacent loudness levels on any given day, it was possible to test the usual $2 \times 2$ (dimension by level) stimulus set at each of three different levels of absolute intensity, while keeping the relative intensity differences fairly constant. Thus, for example, on the first day, an observer might be working with the stimuli generated by the combination of the two pitch levels with Loudness Levels 1 and 2 only. On subsequent days, Loudness Levels 2 and 3 or 3 and 4 would be used.

The third step was a choice reaction time procedure. In all cases, the observer was instructed to discriminate between stimuli on the basis of either pitch or loudness, and respond as quickly as possible by pressing one of the two response buttons. Each of the 18 experimental conditions ( 3 types of stimulus set by 2 dimensions by 3 absolute intensity levels) was administered in blocks of 32 trials twice to each observer. In half of the blocks of one-dimension trials, the irrelevant dimension was held at its higher level, and in the other half it was held at its lower level. Similarly, half of the correlated blocks paired high pitch with high loudness, and the other half paired high pitch with low loudness. Within every block, the level of the relevant dimension varied randomly, with the restriction that each level of that dimension occurred an equal number of times. Before beginning a block, the observer was informed of the relevant dimension and the correct button choice for each of the two levels of that dimension. Button assignments were varied in a haphazard manner between blocks, under the constraint that for every observer, each level was assigned to the left and right button equally often. Each block 
was begun with a sample of four sets (either 2 or 4 stimuli each) of the stimuli to be used in that block, presented in a simple alternating sequence. Following these practice trials, the test trials were begun. Stimuli were presented with an interstimulus interval of $4 \mathrm{sec}$ until the entire block was completed.

An experimental session consisted of six blocks, composed of the combination of two dimensions and three types of stimulus set, with the absolute intensity level held constant at one of its values for the entire session. Each observer participated in six sessions, in which the order of conditions within a session was determined by a balanced Latin square. Absolute intensity level was counterbalanced across sessions (abccba).

\section{RESULTS}

Mean reaction times for all six observers are shown in Table 1. It can be seen that, relative to performance in the one-dimension condition, reaction times were faster in the correlated condition and slower in the orthogonal condition. A factorial analysis of variance indicates that only type of stimulus set had a significant effect, $F(2,10)=$ $170.36, p<.01$. A comparison of means by the Newman-Keuls procedure reveals that both the correlated and orthogonal conditions differed significantly from the one-dimension condition, $\mathrm{p}<.01$. Increases in absolute intensity level seemed to reduce reaction times between the lowest and middle levels, but no further reduction was apparent in moving to the highest intensity level. One-dimension performance at all absolute intensity levels appeared to be better for discriminations of pitch than for those of loudness.

Error rates for all observers ranged from $3.86 \%$ to $5.99 \%$, with a mean of $5.03 \%$. None of the experimental conditions seemed exceptionally conducive to mistakes, and other than a moderate speedaccuracy tradeoff between subjects over all conditions (i.e., subjects with slower overall reaction times committed fewer errors), no trends were evident. There was no further analysis of errors or any special analysis of reaction times for erroneous responses.

\section{DISCUSSION}

It appears that classification speeds in this task were improved by the addition of redundant information and impeded by irrelevant stimulus variation. This pattern of results is typical of that for integral stimulus dimensions (Garner, 1974). Furthermore, it largely agrees with results of a similar experiment in which the loudness and pitch of auditory stimuli were manipulated (Wood, 1975). That experiment also measured discrete reaction times, but only the one-dimension and orthogonal conditions were tested. Nevertheless, the mean difference of $39.4 \mathrm{msec}$ between those conditions compares quite favorably with the corresponding value of $44.5 \mathrm{msec}$ obtained in the present study.
Table 1

Mean Choice Reaction Time (in Milliseconds) of Six Observers for Every Combination of Relevant Dimension, Type of Stimulus Set, and Absolute Intensity Level

\begin{tabular}{lccc}
\hline & \multicolumn{3}{c}{ Type of Stimulus Set } \\
\cline { 2 - 4 } Rimension & One Dimension & Correlated & Orthogonal \\
\hline & \multicolumn{3}{c}{ Absolute Intensity Level 1} \\
Pitch & 430 & 402 & 466 \\
Loudness & 464 & 409 & 524 \\
& \multicolumn{4}{c}{ Absolute Intensity Level 2 } \\
Pitch & 402 & 384 & 428 \\
Loudness & 425 & 394 & 496 \\
& \multicolumn{4}{c}{ Absolute Intensity Level 3} \\
Pitch & 408 & 398 & 427 \\
Loudness & 428 & 390 & 484 \\
& \multicolumn{4}{c}{ Mean } \\
Pitch & 413 & 394 & 440 \\
Loudness & 439 & 397 & 501 \\
\hline
\end{tabular}

Note-Absolute Intensity Level 1 involved stimuli at 22 and $28 \mathrm{~dB}$ sensation level ( $S L$ ), Level 2 at 28 and $34 \mathrm{~dB} S L$, and Level 3 at 34 and $40 \mathrm{~dB}$ SL. Entries under the "Mean" subheading are data collapsed over absolute intensity levels.

According to Garner (1974), these facilitation and interference effects are due to two different processes. The facilitatory effect found with correlated stimulus dimensions is explained as follows: Since integral dimensions are effectively fused into a single stimulus entity, and since stimuli so defined are related to one another by a Euclidean metric, stimuli that differ in two correlated, integral dimensions are more separated in psychological space than stimuli differing along either dimension alone. This increased separation results in greater stimulus discriminability, which is reflected in shorter reaction times.

Interference effects in the orthogonal condition are believed to be related to the common finding that choice reaction time to a stimulus is shorter if the stimulus and response are repeated than if either is different from that of the previous trial (Felfoldy, 1974; Garner, 1974). Furthermore, reaction time is even slower when both the stimulus and response are changed from the trial that came before. Thus, the slower reaction times in the orthogonal condition, as compared with the one-dimension condition, might represent the effect of reducing the frequency of the easier event (stimulus and response repetition) and introducing a substantial proportion of more difficult conditions (stimulus change and response repetition). It might be worth noting that what have been referred to as "changes" in the stimulus, even when those changes are irrelevant to the response (i.e., stimulus change and response repetition), are in fact changes because the integral nature of the stimulus dimensions makes it very difficult to filter or ignore these changes. With separable dimensions, such filtering is possible, and the effective stimulus 
is not changed by irrelevant dimensional variation. Thus with separable dimensions, there is typically no interference effect in the orthgonal condition.

An alternative explanation for reaction time differences across conditions is that these effects represent response selection processes, rather than perceptual processes. Since training and testing procedures required the use of each dimension as the basis for classification, it is possible that both dimensions could influence response tendencies in all conditions. Thus, even with two stimulus dimensions that are perceptually distinct, response biases could induce a pattern of results similar to that expected for integral dimensions. There are, however, several facts that suggest that this is not the case.

The experimental paradigm employed in the present study has been used by several other investigators (Garner, 1974). The first of these investigators, Garner and Felfoldy (1970), found strikingly different patterns of results across one-dimension, correlated, and orthogonal conditions for different pairs of stimulus dimensions. Since the experimental design was identical in all cases, the conclusion is that the different outcomes resulted entirely from the nature of the dimensions tested.

Further evidence against the hypothesis of response selection effects comes from the work of Pomerantz and Garner (1973). This study consisted of a visual speeded classification task performed with a design similar to that used in the present experiment. However, other tests were also run, in which one group of subjects made the classifications only on the basis of one of the stimulus dimensions and another group of subjects classified on the basis of the other dimension. It was found that this modification, one which would seem to prevent the acquisition of response biases, did not alter the pattern of results. It is therefore concluded that those results were not artifacts of the testing method.

A final argument against response artifacts may be drawn from an examination of the data in the present study. When results are analyzed separately for each session, the overall pattern of results, facilitation with correlated dimensions and interference with orthogonal dimensions, is the same throughout the course of the experiment. This analysis does not preclude the acquisition of response biases during practice sessions. However, it is unlikely that biases acquired so quickly would persist unchanged through several days of testing. Furthermore, this stability of results has been found in other experiments using the same paradigm (Garner \& Felfoldy, 1970).

Thus there is strong reason to believe that response selection tendencies are not responsible for the effects in this study, and that differences across conditions do, in fact, result from perceptual processes. Nevertheless, in order to obtain more direct and conclusive support for this interpretation, repetition of the present experiment with other designs, such as one performed with separable stimulus dimensions, may be required.

Although no effects other than those due to type of stimulus set were found to be statistically significant, there is one irregularity in the data that deserves further comment. This concerns the apparent asymmetry of effects between judgments made on the basis of pitch and those made on the basis of loudness. That is, at all absolute intensity levels, both facilitation and interference effects were smaller for discriminations of pitch than for those of loudness. Similar, but more extreme, asymmetries found in the data of Day and Wood (1972) and Wood (1974) have been taken as evidence for serial or serialparallel models of processing. The suggestion is that if processing of one dimension (A) always occurs before processing of the other $(B)$, then dimensional interactions can be avoided when $A$ is the relevant dimension, but not when $B$ is the relevant dimension.

A more plausible explanation for the asymmetric effects found in the present study concerns the relative discriminability of pitch and loudness differences. As noted earlier, one-dimension performance indicated that, overall, pitch was more discriminable than loudness. It seems reasonable, then, that adding the more powerful pitch dimension to stimuli in which loudness is the relevant dimension would lead to larger facilitation or interference effects. Similarly, adding the weaker loudness dimension to stimuli to be judged on the basis of pitch has relatively little effect. This analysis is also consistent with predictions of performance with integral stimulus dimensions. That is, unequally discriminable stimulus dimensions would be expected to generate this pattern of results if they were being governed by a Euclidean metric. It is expected that, in cases in which the one-dimension reaction times are nearly equal, facilitation and interference would be the same whether pitch or loudness was the relevant dimension.

The general conclusion that may be drawn from this study is that, at least in certain tasks, tactile information processing can be improved by redundancy and impeded by irrelevant stimulus variation. These effects may depend on the relative discriminability of the dimension involved. The applicability of these findings to the construction of more effective cutaneous communication systems is still, of course, remote. Nevertheless, it is demonstrated in an elementary way that dimensional interactions can enhance the discriminability of tactile patterns, and that the selection of elements for useful patterns must observe the constraint that irrelevant dimensional variations be reduced to a minimum. Beyond this, there probably exist a large number of effects arising from complex dimensional interactions. In order 
to explore these effects, it will be necessary to extend the present work to a wider variety of stimulus dimensions and experimental tasks.

\section{REFERENCES}

DAY, R. S., \& WooD, C. C. Interactions between linguistic and nonlinguistic processing. Journal of the Acoustical Society of America, 1972, 51, 79.

FarRell, G. Avenues of communication. In P. A. Zahl (Ed.), Blindness: Modern approaches to the unseen envinonment. Princeton, N.J: Princeton University Press, 1950. Pp. 313-345.

FeLfOLdY, G. L. Repetition effects in choice reaction time to multidimensional stimuli. Perception \& Psychophysics, 1974, 15, 453-459.

GARNER, W. R. Information integration and form of encoding. In A. W. Melton \& E. Martin (Eds.), Coding processes in human memory. Washington: Winston, 1972. Pp. 261-281.

GARNER, W. R. The processing of information and structure. Potomac, Md: Erlbaum, 1974.
GARNer, W. R., \& Felfoldy, G. L. Integrality of stimulus dimensions in various types of information processing. Cognitive Psychology, 1970, 1, 225-241.

Geldard, F. A. Tactile communication. In T. A. Sebeok (Ed.), How animals communicate. Bloomington, Ind: University Press, in press.

Pomerantz, J. R., \& Garner, W. R. Stimulus configuration in selective attention tasks. Perception \& Psychophysics, 1973, 14, 565-569.

Woon, C. C. Parallel processing of auditory and phonetic information in speech discrimination. Perception \& Psychophysics, 1974, $15,501-508$.

Woon, C. C. Auditory and phonetic levels of processing in speech perception: Neurophysiological and information-processing analyses. Journal of Experimental Psychology: Human Perception and Performance, 1975, 104, 3-20.

(Received for publication October 11, 1976; revision accepted March 8, 1977.) 